Article

\title{
Analyzing Zone-Based Registration Using a Three Zone System: A Semi-Markov Process Approach
}

\author{
Jihee Jung ${ }^{1}$ and Jang Hyun Baek ${ }^{2, *}$ \\ 1 CAMTIC Advanced Mechatronics Technology Institute, Jeonju 54852, Korea; jjh121004@camtic.or.kr \\ 2 Department of Industrial \& Information Systems Engineering and the RCIT, Jeonbuk National University, \\ Jeonju 54896, Korea \\ * Correspondence: jbaek@jbnu.ac.kr; Tel.: +82-63-270-2330
}

Received: 30 June 2020; Accepted: 10 August 2020; Published: 17 August 2020

\begin{abstract}
The location of user equipment (UE) should always be maintained in order to connect any incoming calls within a mobile network. While several methods of location registration have been proposed, most mobile networks have adopted zone-based registration due to its superior performance. Even though recommendations from research on these zone-based systems state that multiple zones can be stored in a zone-based registration system, actual current mobile networks only employ a zone-based registration system that stores a single zone. Therefore, some studies have been conducted on zone-based registration using multiple zones. However, most of these studies consider only two zones. In this study, through the development of a semi-Markov process approach, we present a simple but accurate mathematical model for zone-based registration using three zones. In addition, our research results in zone-based registration systems where one, two and three zones are used to suggest the optimal management scheme for zone-based registration. Given that most mobile networks have already adopted some kind of zone-based registration, these results are able to directly enhance the performance of the actual mobile network in the near future with the minimum of effort required for implementation.
\end{abstract}

Keywords: location registration; zone-based registration; performance analysis; semi-Markov process; mathematical model

\section{Introduction}

The awareness of the location of user equipment (UE, or mobile phone) should always be maintained so as to be able to connect any incoming calls to that UE anywhere within that mobile network. Location registration is the process by which a UE registers its location and status to a network database (DB) whenever it enters a new location area. If location registration is not performed properly, the network cannot know the location of the UE exactly and may need to page many cells in the network to find its location $[1,2]$.

Many methods of registering location have been proposed, such as movement-based [3-5], timer-based [2], distance-based [2,5-7], zone-based [1,2,8-12] and tracking area list-based [13-19] registration. In particular, several studies on tracking area list-based registration and the corresponding performance improvement have been conducted in recent years [13-19] since current specifications do not provide any details on how to define tracking area lists and allocate them to UEs.

Among the methods mentioned above, most mobile networks have adopted zone-based registration due to its superior performance and ease of implementation. In this kind registration, the mobile network is split into zones consisting of several cells. UE is required to register new location information to the network whenever it moves to a new zone, this is called location registration. When a call arrives on the UE, the network sends paging messages to all of the cells in the UE's zone to identify 
the cell to which the UE belongs and then connects the call. This process is called paging. Generally, as the number of cells in a zone decreases, the registration load increases but paging load decreases. Therefore, the trade-off between the registration and paging load is used to determine the optimal operating scheme for zone-based registration.

Although recommendations for mobile networks state that multiple zones can be stored, actual mobile networks employ a zone-based registration that only stores a single zone (1Z). Due to this some studies have been conducted on zone-based registration that investigated a system with multiple zones. However, most of these studies consider only two zones (2Z) [1,9-11] or have found the results of using three zones (3Z) to be too complicated [12]. In this study, through the development of a semi-Markov process approach, we present a new mathematical model for zone-based registration using three zones. In addition, research results on zone-based registration with one, two and three zones are used to suggest an optimal management scheme for zone-based registration.

The structure of the paper is as follows: Section 2 introduces zone-based registration with multiple zones. Section 3 proposes a new mathematical model based on the semi-Markov process theory, to analyze the accurate performance of the $3 \mathrm{Z}$ system. Section 4 carries out a numerical study to investigate the performance of the zone-based registration with multiple zones. Finally, Section 5 concludes this paper.

\section{Zone-Based Registration}

Let us consider zone-based registration with multiple zones. First, let us take a brief look at zone-based registration using two zones (2Z).

If UE uses two zones, as shown in Figure 1, location registration will not occur if the UE leaves and returns to zone $X$ where it was initially registered. In this case, however, the network knows that the UE is in zone $Y$, where it was registered most recently. Therefore, the network starts paging zone $Y$ first. If there is no response in this zone, it then pages zone $X$ to receive an acknowledgement. As a result, location registration decreases, but paging load increases compared to zone-based registration with one zone (1Z). In order to obtain an accurate paging load, the probability of being in zone $X$, not the most recently registered zone, must be determined [1].

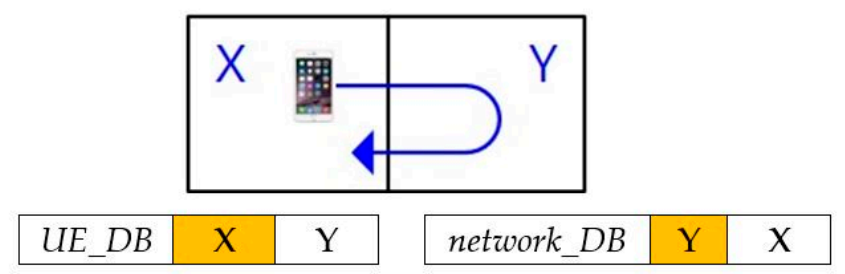

Network cannot know when UE returns to $\mathrm{X}$ since there is no registration process.

Figure 1. Zone-based registration with two zones.

Let us now look at zone-based registration with three zones (3Z), which is the main subject of this study. If the UE uses three zones, as shown in Figure 2, location registration will not occur when the UE leaves and returns to zone $Y$ where it had already been registered. On the other hand, the probability of returning to stored zones ( $\mathrm{X}$ or $\mathrm{Y}$ ) against staying in the most recently registered zone $(\mathrm{Z})$ increases compared to a $2 Z$ system, which increases paging load. As a result, location registration is reduced, but the paging load is increased compared to $1 \mathrm{Z}$ or $2 \mathrm{Z}$ systems.

In this study, a semi-Markov process model was developed to present an accurate mathematical model for a $3 Z$ system. 


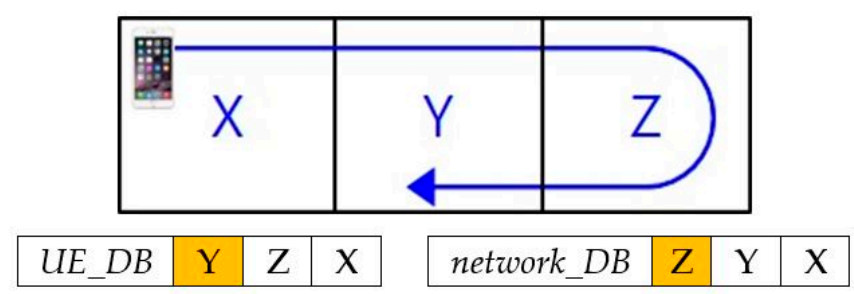

Network cannot know when UE returns to $Y$ since there is no registration process.

Figure 2. Zone-based registration with three zones.

\section{New Mathematical Model}

In this section, we propose a new mathematical model based on a semi-Markov process theory, to analyze the accurate performance of the $3 \mathrm{Z}$ system from the viewpoint of the total signaling cost on radio channels.

\subsection{Notations and Assumptions}

We define the following notations to analyze the signaling cost on radio channels.

- $\mathrm{C}_{\mathrm{p}}$ : Signaling cost for paging per cell on radio channels

- $\mathrm{C}_{\mathrm{u}}$ : Signaling cost for one location registration on radio channels

- $\theta$ : Probability of returning to the registered zone

- $n$ : Number of cells per zone

- $\quad T_{i}$ : Interval between two incoming calls (r.v. (random variable), $\left.T_{i} \sim \operatorname{Exp}\left(1 / \lambda_{i}\right), \mathrm{E}\left(T_{i}\right)=1 / \lambda_{i}\right)$

- $T_{o}$ : Interval between two outgoing calls (r.v., $\left.T_{o} \sim \operatorname{Exp}\left(1 / \lambda_{o}\right), \mathrm{E}\left(T_{o}\right)=1 / \lambda_{o}\right)$

- $\quad T_{c}$ : Interval between two calls (r.v., $\left.T_{\mathcal{c}} \sim \operatorname{Exp}(1 / \lambda), \mathrm{E}\left(T_{\mathcal{c}}\right)=1 / \lambda\right)$

- $\quad T_{m}$ : Sojourn time in a zone (r.v., $\left.\mathrm{E}\left(\mathrm{T}_{m}\right)=1 / \mu\right)$

- $R_{m}$ : Interval between the arrival of the call, and the time when the UE moves out of the zone (r.v.)

- $\rho$ : Incoming calls to mobility ratio $\left(\mathrm{CMR}=\lambda_{i} / \mu=\rho\right)$

- $f_{m}^{*}(s)$ : Laplace-Stieltjes transform for $T_{m}\left(=\int_{t=0}^{\infty} e^{-s t} f_{m}(t) d t\right)$

In addition, we assume the following for square zones, as shown in Figures 1 and 2, in order to analyze the registration load over 3Zs.

- The interval between two calls, $T_{\mathcal{c}}$, follows an exponential distribution with mean $1 / \lambda$.

- The UE's sojourn time in a zone, $T_{m}$, follows a general distribution with mean $1 / \mu$.

- The probability that UE returns to the last zone is $\theta$.

- The probability that UE moves to one of the three zones except the last zone is the same (in other words, $(1-\theta) / 3)$.

- Call holding time is so short that it can be ignored.

Note that, by the additivity of Poisson processes [20], incoming calls with rate $\lambda_{i}$ and outgoing calls with rate $\lambda_{o}$ form a total call rate $\lambda\left(=\lambda_{i}+\lambda_{o}\right)$.

\subsection{Semi-Markov Process Model for $3 Z$}

Many studies on location registration assume that sojourn time in a zone follows an exponential distribution. On the other hand, our model applies to any general distributions for sojourn time in a zone only if its Laplace-Stieltjes transform can be obtained.

Furthermore, many studies employ a symmetric random-walk mobility model that gives the same probability of moving to any of the neighboring zones. However, it is easy to see that these assumptions are not realistic when considering the mobility of the actual UE. Normally, the next zone 
of the UE is related to the current zone. Therefore the assumption of a symmetric random walk is not realistic and does not reflect true UE behavior. Therefore, the probability of returning to the previous zone is assumed to be $\theta$ to reflect the dependency between the current zones and the next zone $[1,10,11]$. In a square zone environment, as shown in Figures 1 and 2, $\theta$ would normally have a value greater than 0.25 . Note that $\theta=0.25$ means a symmetric random walk model.

To illustrate the semi-Markov process model, we take into consideration the composition of the three zones for a $3 Z$ system.

\subsubsection{Definition of State by the Position of Each Zone in a 3Z System}

Among the three zones, let us call the two zones at each end edge zones and the middle zone center zone and mark them as $\mathrm{E}$ and $\mathrm{C}$, respectively. Sometimes, it is simpler to mark the two edge zones as (1) and (3), and the center zone as (2). Note that the edge zone, marked as (1) ( $\left.E_{1}\right)$, is where location registration occurred more recently than the other edge zone $\left(\mathrm{E}_{3}\right)$.

For example, let us assume UE moves in the order of zone $X$ (and registers) $\rightarrow$ zone $Y$ (and registers) $\rightarrow$ zone $Z$ (and registers) $\rightarrow$ zone $Y$, as shown in Figure 3. In this situation, zone $X$ and zone $\mathrm{Z}$ are the edge zones, and zone $\mathrm{Z}$ is where location registration occurred most recently, we define this as state 1 .

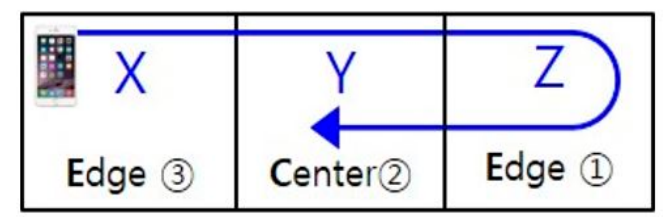

(1) is the edge zone where registration occurred more recently than the other edge zone.

Figure 3. Edge and center zones in a $3 Z$ system.

Let us define the state where we apply our semi-Markov process model. We define the following states with respect to the regular case.

$$
\begin{aligned}
& \mathrm{E}_{i j}(i=1,2,3 ; j=1,2,3) \\
& \mathrm{C}_{i j}(i=1,2,3 ; j=1,2,3)
\end{aligned}
$$

$\mathrm{E}$ and $\mathrm{C}$ indicate that the most recently registered zones are an edge or center zones, respectively. The first subscript $i(=1,2,3)$ represents the UE's immediately previous zone, the second subscript $j(=1,2,3)$ indicates the UE's current zone.

For example, for the case of the UE shown in Figure 3, its state is $E_{12}$ since the current zone is the center $(j=2)$ and the last zone is the edge zone where it registered its location $(i=1)$. If the UE in Figure 3 returns to zone $X$, its state will be $E_{23}$ since the last zone is the center $(i=2)$ and the current zone is the edge zone where it did not register its location most recently $(j=3)$. In addition, if the above $\mathrm{UE}$ with a state of $\mathrm{E}_{23}$ returns to zone $\mathrm{Y}$, its state will be $\mathrm{E}_{32}$ since the last zone is the edge zone where it did not register its location most recently $(i=3)$ and the current zone is the center $(j=2)$.

On the other hand, if the UE in Figure 3 returns to zone $Z$, its state will be $E_{21}$ since the last zone is the center $(i=2)$ and the current zone is the edge zone where it registered its location most recently $(j=1)$.

Figure 4 presents a collection of examples for states $E_{i j}$.

Example for $C_{i j}(i=1,2,3 ; j=1,2,3)$ will be given in the next subsection for better understanding. 


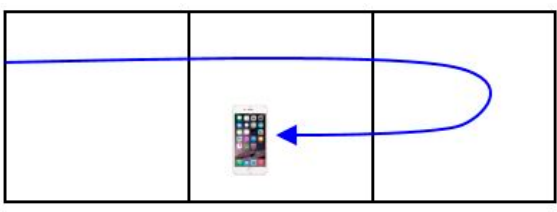

(a) $\mathrm{E}_{12}$

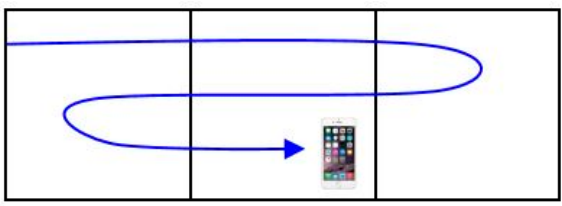

(c) $\mathrm{E}_{32}$

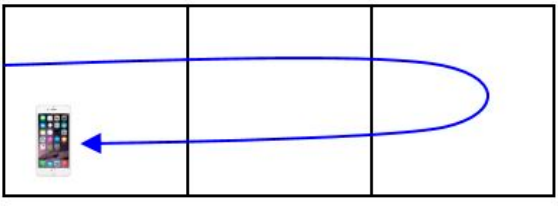

(b) $\mathrm{E}_{23}$

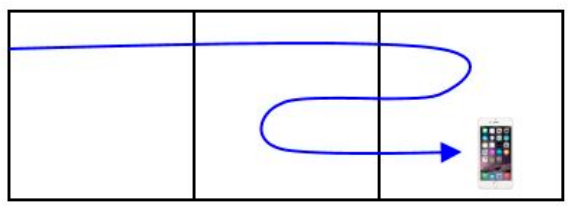

(d) $\mathrm{E}_{21}$

Figure 4. Examples of states $\mathrm{E}_{i j} .:$ (a) $\mathrm{E}_{12} ;$ (b) $\mathrm{E}_{23} ;$ (c) $\mathrm{E}_{32} ;$ (d) $\mathrm{E}_{21}$.

\subsubsection{Definition of States by the Call Occurrence of Each Zone in a 3Z System}

Location registration is divided into two main categories [9-11]. The first category is if the UE moves to a new zone that is not a previously stored zone, then it registers the location. The second category is if a call occurs from/to the UE in the current zone, the network updates the location. In the case of the first category, a location registration message is actually sent to the network. In the case of the second category, a separate location registration message is not delivered to the network, but the network can infer the UE's zone through the call processing messages. The first category is referred to as regular registration (RR) and the second category is referred to as implicit registration (IR).

In the case of the first category, when the UE enters a new zone, the corresponding state is defined and the time it stays in that state is the total sojourn time in that zone. On the other hand, in the case of the second category, when a UE is currently in a zone and a call occurs from/to the UE, the corresponding state is defined so that the time it takes to stay in that state is typically less than the total sojourn time in that zone. In other words, we introduce state I to distinguish cases in the second category from cases in the first category since the time in each state is different.

We define the following states with respect to the second category:

$$
\mathrm{I}_{i j}(i=1,2,3 ; j=1,2)
$$

I indicates that UE receives/generates a call to register its location by IR. The first subscript $i(=1$, $2,3)$ represents the UE's immediately previous zone, the second subscript $j(=1,2)$ indicates the UE's current zone.

It is sufficient to define only states $\mathrm{I}_{21}, \mathrm{I}_{12}$ and $\mathrm{I}_{32}$ concerning call occurrence. For example, if UE with a state of $E_{21}, E_{12}$ and $E_{32}$ receives/generates a call, the UE's zone remains the same but the states are changed to $\mathrm{I}_{21}, \mathrm{I}_{12}$ and $\mathrm{I}_{32}$ respectively.

Figure 5 presents a collection of examples for states $\mathrm{I}_{i j}$.

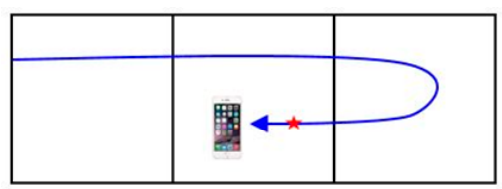

(a) $\mathrm{I}_{12}$

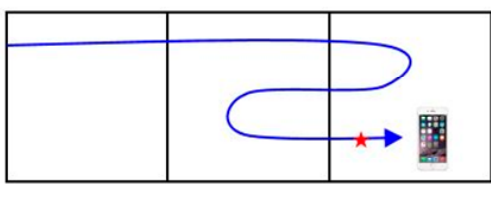

(b) $\mathrm{I}_{21}$

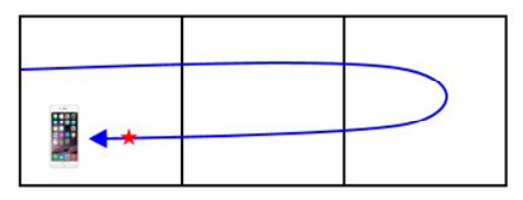

(c) $\mathrm{I}_{32}$

Figure 5. Examples of states $\mathrm{I}_{i j}:$ (a) $\mathrm{I}_{12} ;$ (b) $\mathrm{I}_{21} ;$ (c) $\mathrm{I}_{32}$. 
Note that if $U E$ with a state of $E_{23}$ or $C_{23}$ receives/generates a call, its state will not be $I_{23}$ but $I_{21}$ since the last zone is the center $(i=2)$ and the current zone is the edge zone where it registered its location most recently $(j=1)$ by $I R$, which means that $I_{23}$ is not necessary.

For another example, let us say in the case of the UE shown in Figure 6, its state becomes $\mathrm{I}_{12}$ when a call occurs since its state was $E_{12}$ just before the call occurred. If the UE returns to zone $X$, its state will be $C_{23}$ since the last zone is the center $(i=2)$ and the current zone is the edge zone where it did not register its location most recently $(j=3)$. In addition, if the above UE with a state of $C_{23}$ returns to zone $\mathrm{Y}$, its state will be $\mathrm{C}_{32}$ since the last zone is the edge zone where it did not register its location most recently $(i=3)$ and the current zone is the center $(j=2)$.

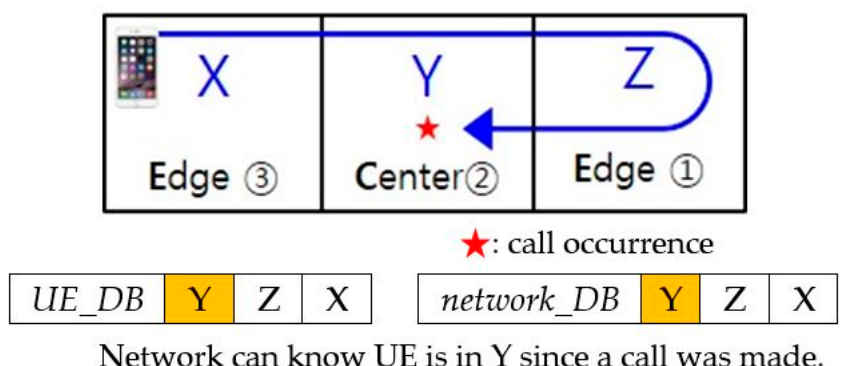

Figure 6. A call occurs in a $3 Z$ system.

On the other hand, if the UE in Figure 6 returns to zone $Z$, its state will be $C_{21}$ since the last zone is the center $(i=2)$ and the current zone is the edge zone where it registered its location most recently $(j=1)$. In addition, if the above $U E$ with a state of $C_{21}$ returns to zone $Y$, its state will be $C_{12}$ since the last zone is the edge zone where it registered its location most recently $(i=1)$ and the current zone is the center $(j=2)$.

Figure 7 presents a collection of examples for states $C_{i j}$.

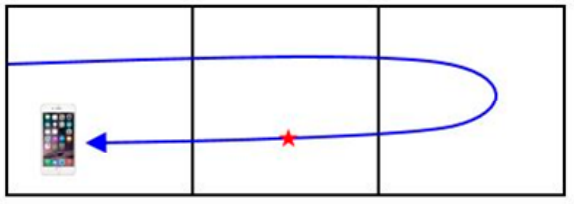

(a) $\mathrm{C}_{23}$

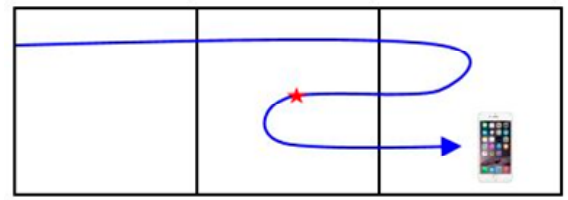

(c) $\mathrm{C}_{21}$

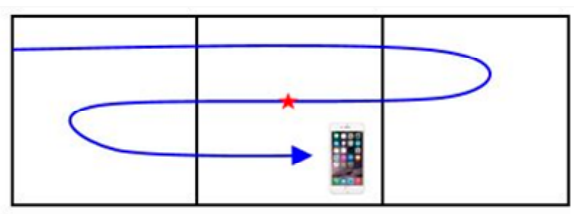

(b) $\mathrm{C}_{32}$

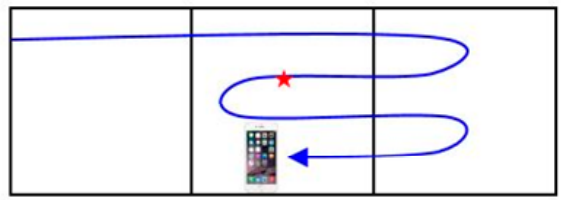

(d) $\mathrm{C}_{12}$

$\star$ : call occurrence.

Figure 7. Examples of states $C_{i j}:$ (a) $C_{23} ;$ (b) $C_{32} ;$ (c) $C_{21} ;$ (d) $C_{12}$.

\subsubsection{Paging Procedure}

In this study, it is assumed that sequential paging to the three zones was performed. When an incoming call occurred, the paging procedure was completed if there was a response from the paged zone. If there was no response, paging was performed again for the remaining zones.

The paging order should be determined to minimize paging costs. In this study, the most recently registered zone is paged first. If there is no response, the center zone is paged next. If there is no response again, the remaining edge zone is paged. Note that if the UE is in state $\mathrm{I}_{i j}$, the first paging attempt always succeeds. 
3.2.4. States for Semi-Markov Process and Transition Diagram

A summary of all the defined states is in Table 1.

Table 1. States for the semi-Markov process model.

\begin{tabular}{ccccc}
\hline State & The Last Zone & Current Zone & Most Recently Registered Zone & No. of Paging \\
\hline $\mathrm{E}_{21}$ & 2 & 1 & edge $(1)$ & 1 \\
$\mathrm{E}_{32}$ & 3 & 2 & edge $(1)$ & 2 \\
$\mathrm{E}_{12}$ & 1 & 2 & edge $(1)$ & 2 \\
$\mathrm{E}_{23}$ & 2 & 3 & edge $(1)$ & 3 \\
$\mathrm{C}_{21}$ & 2 & 1 & center $(2)$ & 2 \\
$\mathrm{C}_{12}$ & 1 & 2 & center $(2)$ & 1 \\
$\mathrm{C}_{32}$ & 3 & 2 & center $(2)$ & 1 \\
$\mathrm{C}_{23}$ & 2 & 3 & center $(2)$ & 3 \\
$\mathrm{I}_{21}$ & 2 & 1 & edge $(1)$ & 1 \\
$\mathrm{I}_{12}$ & 1 & 2 & center $(2)$ & 1 \\
$\mathrm{I}_{32}$ & 3 & 2 & center $(2)$ & 1 \\
\hline
\end{tabular}

The zone registered most recently by the UE can be the edge or center zone as shown in the table above. Additionally, all the states of the UE can be defined by classifying its state with respect to the last and current zones.

If the UE's current zone is an edge zone, regardless of whether or not the edge zone is 1 or 3 , the last zone is unconditionally a center zone. As a result, these states are expressed as $E_{21}$ or $E_{23}$. If the state is $E_{23}$, this means that the UE's most recently registered location is in an edge zone and it moved to the other edge zone that is already in the zone list. If the status is $E_{21}$, this means that the UE's most recently registered location is in an edge zone and it moved to another zone that is already in the zone list and its current zone is an edge zone in which it registered its location most recently.

Through a time-consuming process, a state transition diagram for all the states was obtained, as shown in Figure 8.

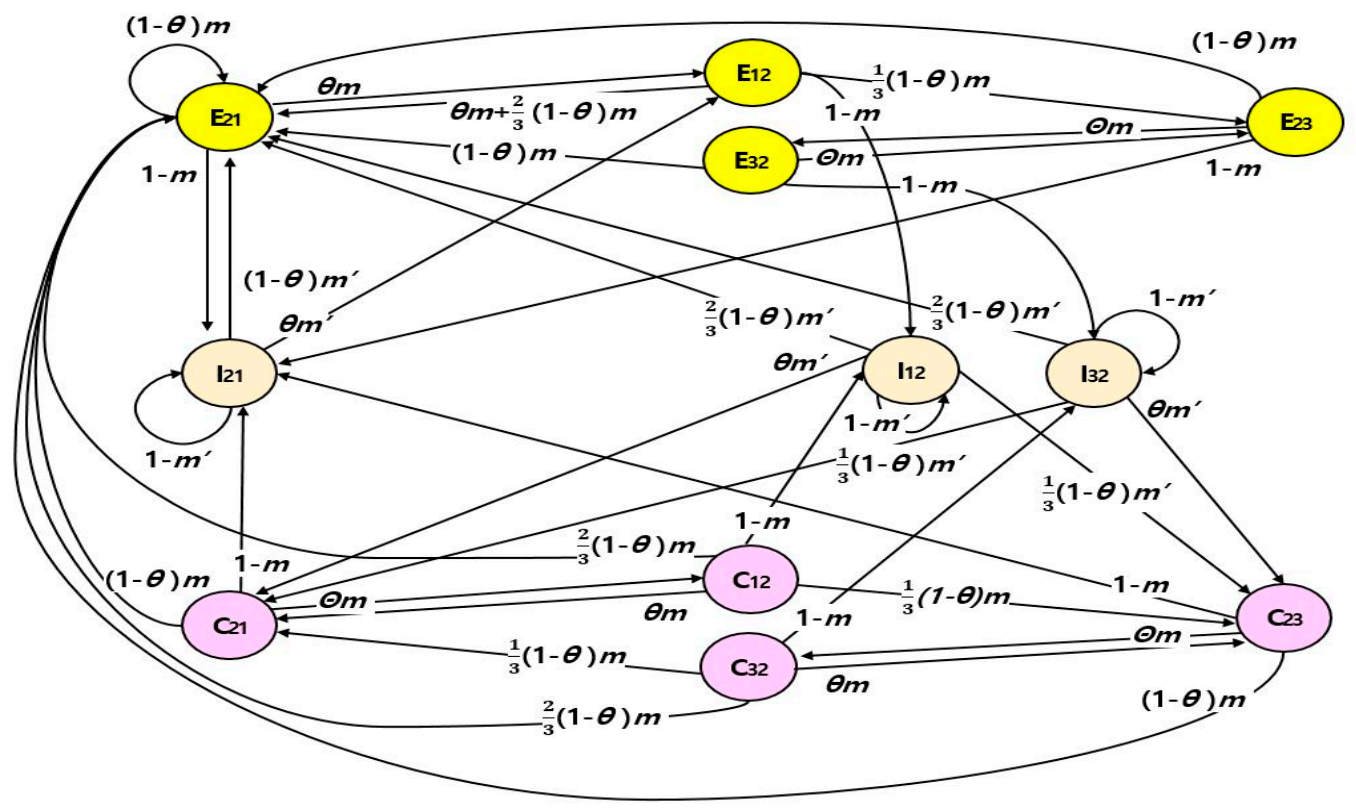

Figure 8. State transition diagram for a $3 Z$ system. 


\subsubsection{Calculation of State Transition Probabilities}

Note that, in the state transition diagram, every transition probability contains $m$ or $m^{\prime}$. The first $m$ is the probability that the UE is in a zone and will move to the neighboring zone before a call to/from the UE occurs. $m$ can be obtained using the following equation:

$$
m=P\left(T_{c}>T_{m}\right)=\int_{t_{m}=0}^{\infty} \int_{t_{c}=t_{m}}^{\infty} \lambda e^{\lambda t_{c}} f_{m}\left(t_{m}\right) d t_{m} d t_{c}=f_{m}^{*}(\lambda)
$$

where $f_{m}^{*}(s)$ is the Laplace-Stieltjes transform for $T_{m}\left(=\int_{t=0}^{\infty} e^{-s t} f_{m}(t) d t\right)$.

Next, let us derive $m^{\prime}=P\left(T_{c}>R_{m}\right)$, which is the probability that the UE, whose state changed due to a call without movement to a new zone, will move to the neighboring zone before another call from/to the UE occurs.

The density function of $R_{m}, f_{r}(t)$ comes from the random observer property [1],

$$
f_{r}(t)=\mu \int_{\tau=t}^{\infty} f_{m}(\tau) d \tau=\mu\left(1-F_{m}(t)\right)
$$

The Laplace-Stieltjes transform for the distribution is as follows:

$$
\begin{gathered}
f_{r}^{*}(s)=\int_{t=0}^{\infty} e^{-s t} f_{r}(t) d t=\int_{t=0}^{\infty} e^{-s t} \mu\left(1-F_{m}(t)\right) d t=\frac{\mu}{s}-\int_{t=0}^{\infty} e^{-s t} \mu F_{m}(t) d t \\
=\frac{\mu}{s}+\left[\frac{\mu}{s} e^{-s t} \mu F_{m}(t)\right]_{t=0}^{\infty}-\frac{\mu}{s} \int_{t=0}^{\infty} e^{-s t} f_{m}(t) d t=\frac{\mu}{s}\left(1-f_{m}^{*}(\lambda)\right)
\end{gathered}
$$

Then, we have:

$$
m^{\prime}=P\left(T_{c}>R_{m}\right)=\int_{r_{m}}^{\infty} \int_{t_{c}=r_{m}}^{\infty} \lambda e^{-\lambda t_{c}} f_{r}\left(r_{m}\right) d t_{c} d r_{m}=\int_{r_{m}=0}^{\infty} f_{r}\left(r_{m}\right) e^{-\lambda t_{c}} d r_{m}=\frac{\mu}{\lambda}\left(1-f_{m}^{*}(\lambda)\right) .
$$

\subsubsection{Calculation of Sojourn Time in Each State}

It is necessary to note that the sojourn time of state $\mathrm{I}_{i j}(i=1,2,3 ; j=1,2,3)$ is different from the sojourn time of state $\mathrm{E}_{i j}(i=1,2,3 ; j=1,2,3)$ or state $\mathrm{C}_{i j}(i=1,2,3 ; j=1,2,3)$. The sojourn time of state $\mathrm{E}_{i j}(i=1,2,3 ; j=1,2,3)$ or state $\mathrm{C}_{i j}(i=1,2,3 ; j=1,2,3)$ is the interval from the time a UE enters a zone until the time it leaves. On the other hand, the sojourn time of state $\mathrm{I}_{i j}(i=1,2,3 ; j=1,2,3)$ is the interval from the time a call to/from a UE occurs in a zone, until the time it leaves.

In the transition diagram, the UE in state $\mathrm{E}_{21}$ (the state that the UE registered when moving from the center zone to an edge zone) can transit to one of three states: (i) If it moves to a new zone (with probability $\left.(1-\theta) P\left(T_{c}>T_{m}\right)=(1-\theta) m\right)$, its state becomes $\mathrm{E}_{21}$ again. (ii) If it returns to the last zone (with probability $\theta P\left(T_{c}>T_{m}\right)=\theta m$ ), its state becomes $E_{12}$. (iii) If a call from/to the UE occurs before the UE moves to a neighboring zone (with probability $P\left(T_{c}<T_{m}\right)=1-P\left(T_{c}>T_{m}\right)=1-m$ ), UE's state changes to state $\mathrm{I}_{21}$.

We should consider the sojourn time of each state to be different, in order to derive an accurate probability of the first paging failure.

Now, let us derive the sojourn time of state $\mathrm{I}_{i j}(i=1,2,3 ; j=1,2,3)$. Since the sojourn time of state $\mathrm{I}_{i j}(i=1,2,3 ; j=1,2,3)$ can be expressed as:

$$
T_{I}= \begin{cases}T_{c}, & \text { if } T_{c} \leq R_{m} \\ R_{m}, & \text { if } T_{c}>R_{m}\end{cases}
$$

and its mean can be derived as follows: 


$$
\begin{aligned}
\tau_{I} & =\int_{0}^{\infty} \int_{0}^{r_{m}} t_{c} f_{c}\left(t_{c}\right) d t_{c} f_{r}\left(r_{m}\right) d r_{m}+\int_{0}^{\infty} \int_{r_{m}}^{\infty} r_{m} f_{c}\left(t_{c}\right) d t_{c} f_{r}\left(r_{m}\right) d r_{m} \\
& =\int_{0}^{\infty}\left[\int_{0}^{r_{m}} t_{c} f_{c}\left(t_{c}\right) d t_{c}+\int_{r_{m}}^{\infty} r_{m} f_{c}\left(t_{c}\right) d t_{c}\right] f_{r}\left(r_{m}\right) d r_{m} \\
& =\int_{0}^{\infty}\left[\frac{1}{\lambda}\left(1-e^{-\lambda r_{m}}\right)\right] f_{r}\left(r_{m}\right) d r_{m}=\frac{1}{\lambda}\left(1-f_{r}^{*}(\lambda)\right) \\
& =\frac{1}{\lambda}\left(1-\frac{\mu}{\lambda}\left[1-f_{m}^{*}(\lambda)\right]\right)
\end{aligned}
$$

Next, let us derive the sojourn time of state $\mathrm{E}_{i j}(i=1,2,3 ; j=1,2,3)$ or state $C_{i j}(i=1,2,3 ; j=1,2$, 3). Since the sojourn time of state $\mathrm{E}_{i j}(i=1,2,3 ; j=1,2,3)$ or state $C_{i j}(i=1,2,3 ; j=1,2,3)$ can be expressed as:

$$
T_{E}= \begin{cases}T_{\mathcal{c}}, & \text { if } T_{c} \leq T_{m} \\ T_{m}, & \text { if } T_{c}>T_{m}\end{cases}
$$

and its mean can be derived as follows:

$$
\begin{gathered}
\tau_{E}=\tau_{C}=\int_{0}^{\infty} \int_{0}^{t_{m}} t_{c} f_{c}\left(t_{c}\right) d t_{c} f_{m}\left(t_{m}\right) d t_{m}+\int_{0}^{\infty} \int_{t_{m}}^{\infty} t_{m} f_{c}\left(t_{c}\right) d t_{c} f_{m}\left(t_{m}\right) d t_{m}= \\
\int_{0}^{\infty}\left[\int_{0}^{t_{m}} t_{c} f_{\mathcal{c}}\left(t_{c}\right) d t_{c}+\int_{t_{m}}^{\infty} t_{m} f_{c}\left(t_{c}\right) d t_{c}\right] f_{m}\left(t_{m}\right) d t_{m}=\int_{0}^{\infty}\left[\frac{1}{\lambda}\left(1-e^{-\lambda t_{m}}\right)\right] f_{m}\left(t_{m}\right) d t_{m}= \\
\frac{1}{\lambda}\left(1-f_{m}^{*}(\lambda)\right)
\end{gathered}
$$

\subsubsection{Calculation of Steady-State Probabilities}

To get the steady-state probability $\tilde{\pi}$ considering the different sojourn times, we first calculate the steady-state probability $\pi$ for the usual Markov chain with transition probability $\boldsymbol{P}$. This can be obtained by using the following balanced equations [20]:

$$
\pi \boldsymbol{P}=\pi, \quad \sum_{i} \sum_{j}\left(\pi_{C i j}+\pi_{E i j}+\pi_{I i j}\right)=1
$$

Then, the final steady-state probability of the semi-Markov process can be obtained as below [6]:

$$
\begin{aligned}
& \widetilde{\pi}_{C i j}=\frac{\pi_{C i j} \tau_{C}}{\sum_{i} \sum_{j}\left(\pi_{C i j} \tau_{C}+\pi_{E i j} \tau_{E}+\pi_{I i j} \tau_{I}\right)} \\
& \widetilde{\pi}_{E i j}=\frac{\pi_{E i j} \tau_{E}}{\sum_{i} \sum_{j}\left(\pi_{C i j} \tau_{C}+\pi_{E i j} \tau_{E}+\pi_{I i j} \tau_{I}\right)} \\
& \widetilde{\pi}_{I i j}=\frac{\pi_{I i j} \tau_{I}}{\sum_{i} \sum_{j}\left(\pi_{C i j} \tau_{C}+\pi_{E i j} \tau_{E}+\pi_{I i j} \tau_{I}\right)}
\end{aligned}
$$

\subsection{Registration Cost}

By making the assumption that $U$ is the registration cost of one registration, the registration cost between two incoming calls, $\mathrm{Cu}$, can be obtained as follows:

$$
\begin{aligned}
C_{U}=U & \cdot \frac{\mu}{\lambda_{i}} \cdot\left[\widetilde{\pi}_{I 21}(1-\theta) m^{\prime}+\left(\widetilde{\pi}_{I 32}+\widetilde{\pi}_{I 12}\right) \frac{2}{3}(1-\theta) m^{\prime}\right. \\
& +\left(\widetilde{\pi}_{E 21}+\widetilde{\pi}_{C 21}+\widetilde{\pi}_{E 23}+\widetilde{\pi}_{C 23}\right)(1-\theta) m \\
& \left.+\left(\widetilde{\pi}_{E 12}+\widetilde{\pi}_{E 32}+\widetilde{\pi}_{C 12}+\widetilde{\pi}_{C 32}\right) \frac{2}{3}(1-\theta) m\right]
\end{aligned}
$$

\subsection{Paging Cost}

As described in Section 3.2, it is assumed that three-step sequential paging to the three zones was performed. The most recently registered zone is paged first. If there is no response, the center zone is paged next. If there is no response again, the remaining edge zone is paged.

Let us consider the paging cost of a $3 Z$ system when the above three-step sequential paging is applied. Assuming $V$ to be the paging cost for one cell, the paging cost between two incoming calls, $C_{P}$, can be obtained as follows: 


$$
\begin{aligned}
C_{P} & =V \cdot(n \cdot P[1 \text { st paging success }]+2 n \cdot P[2 \text { nd paging success }]+3 \cdot P[3 \text { rd paging success }]) \\
& =V \cdot\left[n\left(\widetilde{\pi}_{E 21}+\widetilde{\pi}_{I 21}+\widetilde{\pi}_{I 32}+\widetilde{\pi}_{I 12}+\widetilde{\pi}_{C 12}+\widetilde{\pi}_{C 32}\right)+2 n\left(\widetilde{\pi}_{E 12}+\widetilde{\pi}_{E 32}+\widetilde{\pi}_{C 21}\right)\right. \\
& \left.+3 n\left(\widetilde{\pi}_{E 23}+\widetilde{\pi}_{C 23}\right)\right] \\
& =V \cdot\left[2 n-n \cdot\left(\widetilde{\pi}_{E 21}+\widetilde{\pi}_{C 21}+\widetilde{\pi}_{I 21}+\widetilde{\pi}_{I 32}+\widetilde{\pi}_{I 12}\right)+n \cdot\left(\widetilde{\pi}_{E 23}+\widetilde{\pi}_{C 23}\right)\right]
\end{aligned}
$$

\subsection{Total Cost}

As a result, the final total cost between two incoming calls can be obtained by adding the paging cost to the registration cost.

$$
T C_{3 Z}=C_{U}+C_{P}
$$

\section{Numerical Results}

We obtained numerical results for $1 \mathrm{Z}, 2 \mathrm{Z}$ and $3 \mathrm{Z}$ systems and compared their performances. We assumed the following:

- The number of cells in a zone, $n$, is 4 .

- The probability of returning to the last zone, $\theta$, is 0.4 .

- Registration cost of one registration, $U$, is 4 and paging cost for one cell, $V$, is $1(U=4, V=1)$.

- Incoming and outgoing call rates are both 1 call per hour $\left(\lambda_{i}=\lambda_{o}=1\right)$.

- UE's sojourn time in a zone, $T_{m}$, follows an exponential distribution with mean $1 / 4(\mu=4)$.

The probability of returning to the last zone, $\theta$, is chosen arbitrarily so that it is larger than 0.25 (symmetric random walk) referring to other research results [1,9-12].

\subsection{Total Cost for Various CMRs}

Figure 9 shows the total costs of $1 \mathrm{Z}, 2 \mathrm{Z}$ and $3 \mathrm{Z}$ systems for various call-to-mobility $\left(\mathrm{CMR}=\lambda_{i} / \mu\right)$ ratios. $C M R=1 / 2$ indicates that the UE entered two zones between incoming calls. Note that a smaller CMR means that the UE entered more zones between incoming calls. The CMR value is considered from 0.125 to 1.00 to consider real situations. From the figure, we can see that $3 Z R$ outperforms not only $1 \mathrm{Z}$ but also $2 \mathrm{Z}$. We can also see that, as CMR decreases, the relative gap between $1 \mathrm{Z} / 2 \mathrm{Z}$ and $3 \mathrm{Z}$ increases. Even if $3 Z$ is expected not to outperform $1 Z / 2 Z$ especially when CMR is very high, such a high CMR seems to be unrealistic. In summary, $3 Z R$ generally outperforms $1 Z$ and $2 Z$ in real situations.

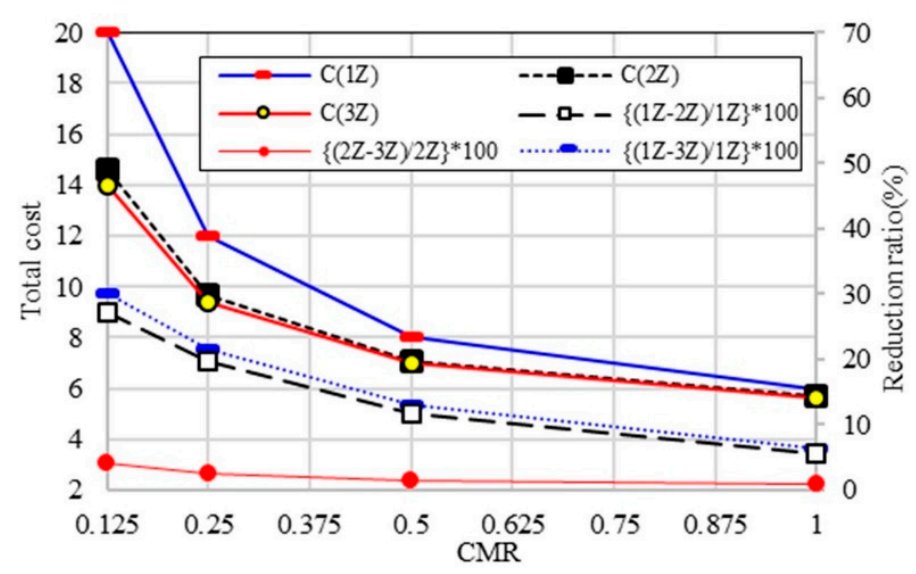

Figure 9. Total cost for various CMR.

\subsection{Total Cost for Various Probabilities of Moving Back to the Last Zone}

Figure 10 shows the total cost for various probabilities of moving back to the last zone, $\theta$, when $\mathrm{CMR}=1$ and $n=4$. The total cost of $2 Z$ and $3 Z$ systems decrease as $\theta$ increases but the total cost of the $3 Z$ system decreases more than that of a $2 Z$ system in our environment, as clearly shown in Figure 10. 
From the figure, we can see that, noting that $\theta$ is generally greater than or equal to 0.25 in a square zone environment, $3 Z$ s are optimal in most cases.

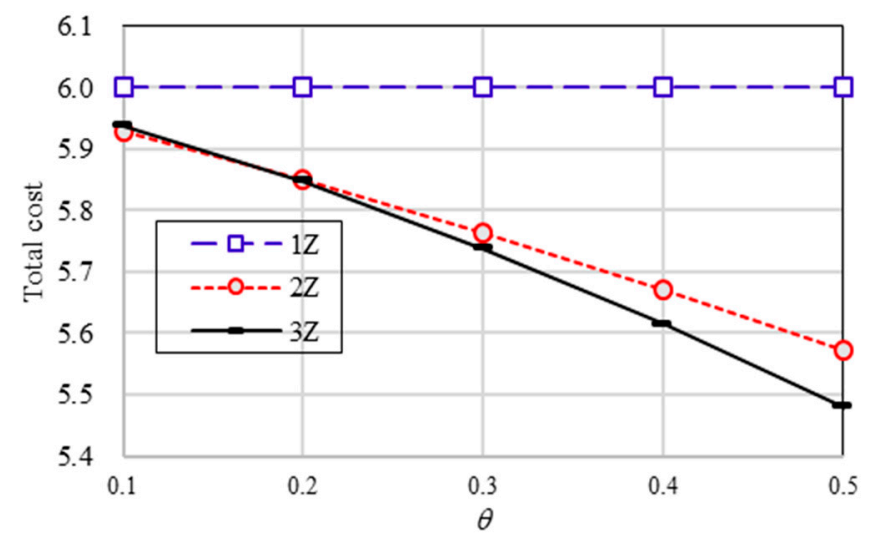

Figure 10. Total cost for various $\theta s$.

\subsection{Total Cost for Various Gamma Distributions}

Let us consider the case where the UE's sojourn time in a zone, $T_{m}$, follows a gamma distribution with a mean of 1 and focus on the comparison between $2 Z$ and $3 Z$ systems since they are both far superior to a $1 Z$ system.

Assuming $\theta=0.3$, Figure 11 compares the performance results for various CMRs and variances. The figure shows that the $3 \mathrm{Z}$ system is superior to the $2 \mathrm{Z}$ system for small CMRs. We can also see that, as the variance decreases, this tendency becomes more definite. Note that, from the figure, the smaller the CMR and the larger the variance, the better the $3 \mathrm{Z}$ system is compared to the $2 \mathrm{Z}$ system but the difference is very small (about $1 \%$ ).

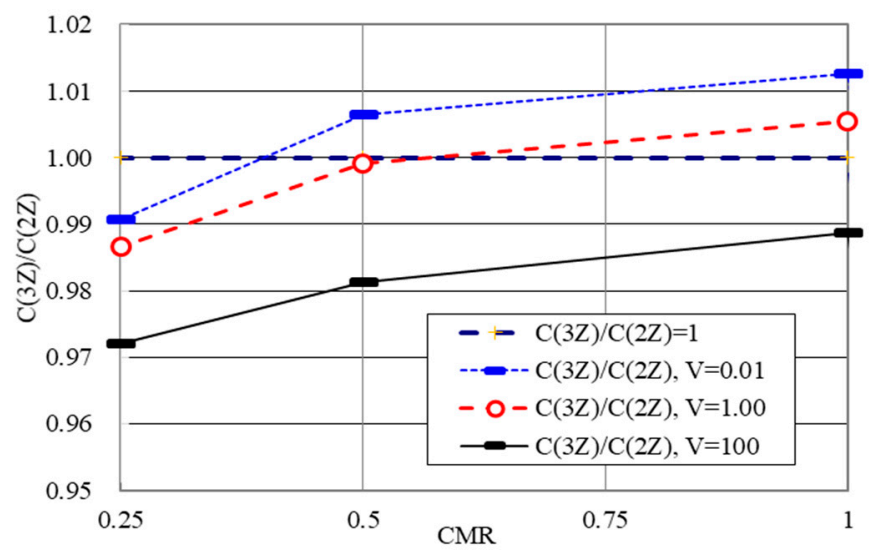

Figure 11. Total cost for various gamma distributions $(\theta=0.3)$.

\subsection{Effect of Implicit Registration by Outgoing Calls}

As explained in Section 3, when a call is made from the UE in the current zone, the network updates the location through the call processing messages without location registration messages, which is called an implicit registration (IR) by an outgoing call. In this case, the paging cost can be reduced in $2 Z$ or $3 Z$ systems since the network can infer the UE's current zone.

Let us examine the IR effect by outgoing calls in a $3 Z$ system numerically. Figure 12 compares the performance results for a $3 Z$ system without IR, a $3 Z$ system with IR and a $1 Z$ system for some CMRs, while assuming $\mathrm{T}_{m}$ follows an exponential distribution with a mean of 1 . In this case, it is also assumed that the outgoing call rate is equal to the incoming call rate $\left(\lambda_{i}=\lambda_{o}\right)$. In the figure, we can see that the $3 Z$ system with IR is much superior to a $3 Z$ system without IR. Note that when IR is not 
considered, the $3 Z$ system is inferior to the $1 Z$ system but when IR is considered, the $3 Z$ is superior to the $1 Z$ system. In conclusion, the IR effect by outgoing calls should be considered for the $3 Z$ system to achieve a minimal total cost.

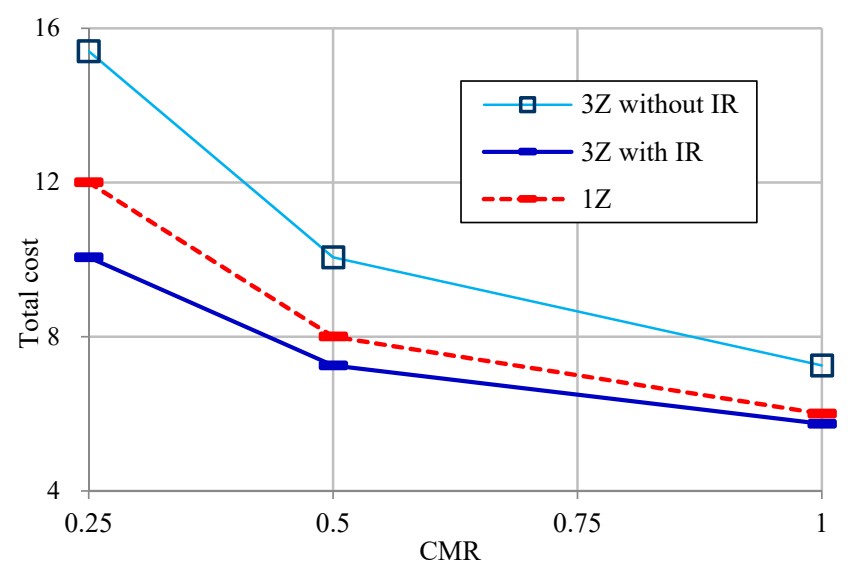

Figure 12. Effect of implicit registration by outgoing calls $(\theta=0.3)$.

\subsection{Effects of the Number of Cells in a Zone and the Registration Cost of One Registration}

In this section, the number of cells in a zone, $n$, is assumed to be 4 . The number of cells in a zone (n) directly affects the paging $\operatorname{cost} C_{P}$. As $n$ increases, $C_{P}$ increases proportionally. From the viewpoint of $3 Z$, the increase of $n$ may degrade the performance of $3 Z$ since the paging $\operatorname{cost} C_{P}$ increases steeply compared to $1 Z$ or $2 Z$. As a result, if $n$ is far greater than $4,3 Z$ may show worse performance than $2 Z$. On the other hand, if $n$ is far less than 4, 3Z may show better performance than $2 Z$.

Note that this kind of scalability can be applied to the other parameters in the similar way. For example, let us consider registration cost. In this section, the registration cost of one registration, $U$, is assumed to be 4 . $U$ directly affects the registration cost $C_{U}$. As $U$ increases, $C_{U}$ increases proportionally. From the viewpoint of $3 Z$, the increase of $U$ may upgrade the performance of $3 Z$ since the location registration $\operatorname{cost} C_{U}$ increases moderately compared to $1 \mathrm{Z}$ or $2 \mathrm{Z}$. As a result, if $U$ is far greater than $4,3 Z$ may show better performance than $2 Z$. On the other hand, if $U$ is far less than 4 , $3 Z$ may show worse performance than $2 Z$.

\section{Conclusions}

In this study, we proposed a very simple new mathematical model using semi-Markov process theory for zone-based registration using three zones (3Z) and analyzed the performance of a $3 Z$ system using the proposed model. The numerical results for the various situations showed that, when the CMR is small, the $3 Z$ system outperforms $1 Z$ and $2 Z$ systems. On the other hand, when the CMR is very large, the $3 Z$ is not much different from $1 Z$ or $2 Z$ systems or is sometimes inferior to $1 Z$ and $2 Z$ systems. In conclusion, depending on the system situation, the optimal management scheme for zone-based registration will change dynamically, so it is necessary to operate zone-based registration flexibly by considering the system situation.

Using a simple mathematical model based on semi-Markov process theory, this study can easily support the prompt determination of the optimal management scheme for zone-based registration. These results are directly able to enhance the performance of actual mobile networks and can be implemented quickly and easily. Since no hardware modifications are necessary to implement a $3 Z$ system, it can be easily applied by modifying the 4G/5G networks of the existing $1 \mathrm{Z}$ based networks. Noting that a $3 Z$ system outperforms a $2 Z$ system in specific conditions, it is possible to adopt an adaptive scheme to dynamically switch between $1 \mathrm{Z}, 2 \mathrm{Z}$ or $3 \mathrm{Z}$ systems according to the current situation. 
Author Contributions: Conceptualization and validation, J.J. and J.H.B.; formal analysis, data curation, J.J.; writing, supervision, project administration, funding acquisition, J.H.B. All authors have read and agreed to the published version of the manuscript.

Funding: This research was supported by the Research Base Construction Fund Support Program funded by Jeonbuk National University in 2019, and the Basic Science Research Program through the National Research Foundation of Korea (NRF) funded by the Ministry of Education (2016R1D1A1B01014615).

Conflicts of Interest: The authors declare no conflict of interest.

\section{References}

1. Lin, Y.B. Reducing location update cost in a PCS network. IEEE/ACM Trans. Netw. 1997, 5, 25-33.

2. 3GPP2 C.S0005-E Version 3.0, Upper Layer (Layer 3) Signaling Standard for cdma2000 Spread Spectrum Systems. June 2011. Available online: http://www.3gpp2.org/Public_html/Specs/C.S0005-E_v3.0_cdma2000_ 1x_Layer-3_20110620.pdf (accessed on 17 August 2020).

3. Li, J.; Kameda, H.; Li, K. Optimal dynamic mobility management for PCS networks. IEEE/ACM Trans. Netw. 2000, 8, 319-327.

4. Wang, X.; Lei, X.; Fan, P.; Hu, R.; Horng, S. Cost analysis of movement-based location management in PCS networks: An embedded Markov chain approach. IEEE Trans. Veh. Technol. 2014, 63, 1886-1902. [CrossRef]

5. Mao, Z.; Douligeris, C. A location-based mobility tracking scheme for PCS networks. Comput. Commun. 2000, 23, 1729-1739. [CrossRef]

6. Baek, J.H.; Lee, T.H.; Kim, J.S. Performance analysis of 2-location distance-based registration in mobile communication networks. IEICE Trans. Commun. 2013, E96-B, 914-917. [CrossRef]

7. Seo, K.H.; Baek, J.H.; Eom, C.S.; Lee, W. Optimal management of distance-based location registration using embedded Markov chain. Int. J. Distrib. Sens. Netw. 2020, 16. [CrossRef]

8. Baek, J.H.; Ryu, B.H.; Lim, S.K.; Kim, K.S. Mobility model and performance analysis for zone-based registration in CDMA mobile communication system. Telecommun. Syst. 2000, 14, 13-30. [CrossRef]

9. Jang, H.S.; Hwang, H.; Jun, K.P. Modeling and analysis of two-location algorithm with implicit registration in CDMA personal communication network. Comput. Ind. Eng. 2001, 41, 95-108. [CrossRef]

10. Baek, W.M.; Yoon, J.H.; Kim, C.S. Modeling and analysis of mobility management in mobile communication networks. Sci. World J. 2014, 2014, 250981. [CrossRef] [PubMed]

11. Baek, J.H. Analyzing zone-based registration in mobile cellular networks. IEICE Trans. Commun. 2017, E100-B, 2070-2078. [CrossRef]

12. Jung, J.; Baek, J.H. Modeling and cost analysis of zone-based registration in mobile cellular networks. ETRI J. 2018, 40, 736-744. [CrossRef]

13. Liou, R.H.; Lin, Y.B. Mobility management with the central-based location area policy. Comput. Netw. 2013, 57, 847-857. [CrossRef]

14. Lin, Y.B.; Liou, R.H.; Chang, C.T. A dynamic paging scheme for long-term evolution mobility management. Wirel. Commun. Mob. Comput. 2015, 15, 629-638. [CrossRef]

15. Deng, T.; Wang, X.; Fan, P.; Li, K. Modeling and performance analysis of a tracking-area-list-based location management scheme in LTE networks. IEEE Trans. Veh. Technol. 2016, 65, 6417-6431. [CrossRef]

16. Jung, J.; Baek, J.H. Reducing paging cost of tracking area list-based mobility management in LTE network. J. Supercomput. 2018. [CrossRef]

17. Alsaeedy, A.A.R.; Chong, E.K.P. Tracking area update and paging in 5G networks: A survey of problems and solutions. Mob. Netw. Appl. 2019, 24, 578-595. [CrossRef]

18. Chen, L.; Liu, H.L.; Fan, Z.; Xie, S.; Goodman, E.D. Modeling the tracking area planning problem using an evolutionary multi-objective algorithm. IEEE Comput. Intell. Mag. 2017, 12, 29-41. [CrossRef]

19. Alsaeedy, A.A.R.; Chong, E.K.P. Mobility management for 5G IoT devices: Improving power consumption with lightweight signaling overhead. IEEE Internet Things J. 2019, 6, 8237-8247. [CrossRef]

20. Ross, S. Introduction to Probability Models, 11th ed.; Elsevier: Cambridge, MA, USA, 2014.

(C) 2020 by the authors. Licensee MDPI, Basel, Switzerland. This article is an open access article distributed under the terms and conditions of the Creative Commons Attribution (CC BY) license (http://creativecommons.org/licenses/by/4.0/). 